\title{
Innovation as part of a wider behavioural syndrome in the guppy: The effect of sex and body size
}

\section{Monica Anderson Berdal ${ }^{1,2}$ (i) | Gunilla Rosenqvist ${ }^{1}$ | Jonathan Wright ${ }^{1}$}

${ }^{1}$ Center for Biodiversity Dynamics (CBD), Department of Biology, Norwegian University of Science and Technology (NTNU), Trondheim, Norway

${ }^{2}$ Department of Biological Sciences, North Dakota State University, Fargo, North Dakota

\section{Correspondence}

Monica Anderson Berdal, Center for Biodiversity Dynamics (CBD), Department of Biology, Norwegian University of Science and Technology (NTNU), Trondheim, Norway.

Email: m.anderson.berdal@gmail.com

Funding information

Research Council of Norway, Grant/Award Number: 223257

Editor: L. Fusani

\begin{abstract}
Recent work on animal personalities has shown that individuals within populations often differ consistently in various types of behaviour and that many of these behaviours correlate among individuals to form behavioural syndromes. Individuals of certain species have also been shown to differ in their rate of behavioural innovation in arriving at novel solutions to new and existing problems (e.g., mazes, novel foods). Here, we investigate whether behaviours traditionally studied in personality research are correlated with individual rates of innovation as part of a wider behavioural syndrome. Guppies (Poecilia reticulata) of both sexes from three different wild population sources were assessed: (a) exploration of an open area; (b) speed through a three-dimensional maze; (c) investigation of a novel object; and (d) attraction to a novel food. The covariance structure (syndrome structure) was examined using structural equation modelling. The best model separated behaviours relating to activity in all contexts from rates of exploration/investigation and innovation. Innovative behaviour (utilizing new food and moving through a novel area) in these fish therefore forms part of the same syndrome as the traditional shy-bold continuum (exploration of an open area and investigation of a novel object) found in many animal personality studies. There were no clear differences in innovation or syndrome structure between the sexes, or between the three different populations. However, body size was implicated as part of the behavioural syndrome structure, and because body size is highly correlated with age in guppies, this suggests that individual behavioural differences in personality/innovation in guppies may largely be driven by developmental state.
\end{abstract}

\section{KEYWORDS}

animal personality, behavioural flexibility, behavioural syndromes, developmental state, habituation, maze exploration

\section{1 | INTRODUCTION}

Animals are often faced with novel environments and contexts and must respond to these challenges with new or modified behavioural solutions to cope with the new conditions (Reader \& Laland, 2003b). This is known as innovation and has been defined by Ramsey, Bastian, and Schaik (2007) as "...the process that generates in an individual a novel learned behaviour that is not simply a consequence of social learning or environmental induction." This separates the origin of a new behaviour from the social spread of the behaviour through a population, that is via cultural transmission (Hoppitt \& Laland, 2013). Innovation is widespread throughout many taxa (Reader \& Laland, 2003b), but it is not clear whether and why individuals differ in their propensity to innovate. In addition, what behaviours are considered as innovative seems to vary across different areas of study. Several studies of innovation in primates and birds often include tasks that demands a higher level of cognition, such as tool use and solving manmade physical puzzles (Goodall, 1964; Rutz \& St Clair, 2012). However, "simpler" tasks, such as learning to utilize a new food source (without the use of tools), are also considered an innovative behaviour. 
This includes milk bottle top opening by blue tits, Cyanistes caeruleus, (Fisher \& Hinde, 1949), and ring-tailed lemurs (Lemur catta) learning to reach water by dipping their tails in a lake and then licking the water off their tail, thereby avoiding predators on the ground and in the water (Hosey, Jacques, \& Pitts, 1997). In this study, we consider any new or modified behaviour used in a novel context as innovative behaviours.

Research on animal personalities has shown that individuals within the same population show consistent differences in behaviour and that these differences are maintained across environmental contexts (Carere \& Maestripieri, 2013; Réale, Dingemanse, Kazem, \& Wright, 2010). Individual differences in a range of different behaviours have also been shown to covary among individuals in what are known as "behavioural syndromes" (Réale, Dingemanse, et al., 2010). For example, bolder individuals tend to be more aggressive than shy individuals, and more exploratory individuals are often more active than less exploratory individuals (Riechert \& Hedrick, 1993; Verbeek, Boon, \& Drent, 1996). If individuals consistently differ in their rate of innovation, innovative behaviours should also be considered as a personality trait (in the broad sense, Réale, Dingemanse, et al., 2010). This has been shown in both great tits (Parus major) and blue tits (Cyanistes caeruleus), where individuals differed consistently in their ability to solved different artificial foraging tasks (MorandFerron, Cole, Rawles, \& Quinn, 2011). Innovation could also possibly be linked to individual differences in other behaviours traditionally studied in personality research, such as boldness and neophilia (Reader \& Laland, 2003b). A link between behavioural type and cognitive type has already been discussed by Sih and Del Giudice (2012), specifically regarding the idea of a speed-accuracy trade-off in foraging. In addition, innovative behaviours involve novel contexts not before experienced by an individual, and so innovation might have risks and/or costs associated with it. This could include increased predation risk, the risk of consuming hazardous foods (Reader \& Laland, 2003b) and reduced efficiency when utilizing familiar resources. Therefore, bold or more risk-taking individuals might be more innovative than shy or less risk-taking individuals, because they approach and investigate unfamiliar resources at a higher rate (Griffin, Guillette, \& Healy, 2015; Reader \& Laland, 2003b; Sih \& Del Giudice, 2012). Such individuals will experience more novel situations in which new and flexible behaviour might be useful and carry some adaptive advantage. However, slower, more thoroughly exploring individuals tend to gather more information about their surroundings and to show greater flexibility in their behaviour (Guillette, Reddon, Hoeschele, \& Sturdy, 2011; Mathot, Wright, Kempenaers, \& Dingemanse, 2012). Thus, while bold individuals might be more likely to be the innovators, it is the shy individuals that seem best suited to the task of social learning needed for the cultural transmission of successful innovative behaviours (Reader \& Laland, 2003b). Although there have been several studies investigating when an individual should copy others (Templeton \& Giraldeau, 1995), and when to rely on private versus public information (van Bergen, Coolen, \& Laland, 2004), few studies have investigated whether some individuals are more prone to solve problems while other individuals are more prone to copy behaviours, leading to the spread of the new behaviour through the population. In addition, few studies have investigated if there is a link between traditional animal personalities and information use (but see Harcourt, Biau, Johnstone, \& Manica, 2010). However, there have been studies showing a link between boldness and flexibility of behaviours. Guillette, Reddon, Hurd, and Sturdy (2009) found that bolder and more exploratory black-capped chickadees (Poecile atricapillus) that enter a novel environment faster were also quicker to learn in an acoustic discrimination task, here used as a measure of cognition, as compared with shyer individuals. However, fast-exploring birds have also been shown to be less flexible in a reversal test, where they learned the change between cue and reward slower compared with slow-exploring individual (Guillette et al., 2011). Similarly, a study of Indian mynas (Acridotheres tristis) found that more innovative individuals-here measured as time to complete an extractive foraging task-were slower to change their behaviour when there was a change in the significance of the food cue (Griffin, Guez, Lermite, \& Patience, 2013). This is consistent with other studies showing that bold proactive individuals are less flexible than shy reactive individuals (Benus, Koolhaas, \& Oortmerssen, 1988; Verbeek, Drent, \& Wiepkema, 1994). In addition, dominant individuals might be more aggressive than subordinates, and as such they could afford to be less innovative and thus avoid any inherent risks, and instead use their social access to limited resources to take advantage of the discoveries by low-ranking individuals in something akin to a producer-scrounger game (Katzir, 1982; Liker \& Barta, 2002). However, there have been very few studies explicitly on the link between performance in personality assays and tests of innovation (but see: Matzel et al., 2006, Light, Kolata, Hale, Grossman, \& Matzel, 2008).

Many studies of animal personality are methodologically quite similar to studies investigating innovation. Boldness is often measured as the time an animal takes to emerge from a refuge or the latency before approaching a novel object (Niemela, Vainikka, Hedrick, \& Kortet, 2012; Verbeek et al., 1994). Approaching a novel area, object or food is therefore equivalent to tests of neophobia versus neophilia, an important component of innovation (Reader \& Laland, 2003b; Webster \& Lefebvre, 2001). In a study by Dingemanse et al. (2007) on threespined sticklebacks (Gasterosteus aculeatus), the response to novel food was classified as an exploratory behaviour, which is very similar to tests in much of the literature concerning innovative behaviour, that is the ability to utilize novel food sources (Reader \& Laland, 2003b). Conversely, many studies on innovation may represent equally good studies of animal personality behaviours. For example, in a study by Laland and Reader (1999a), innovation was measured as the latency before guppies (Poecilia reticulata) went through a maze, where on the other side, they were presented with a novel food. Individuals that were able to figure out the maze fastest and ate the novel food first were classified as more innovative than the slower individuals. However, they could just as easily have been classified as bolder or more exploratory individuals, or just more active. Thus, measures of innovation appear to be confounded or to covary with several aspects of other behaviours currently studied in the context of animal personality. Research is clearly needed that investigates the role of such innovative behaviours in the context of animal personality assays, therefore making it possible to examine the structure of possibly wider behavioural syndromes of individual boldness versus shyness.

In this study, we investigate behavioural syndrome structures in guppies, specifically to see whether innovative behaviours are part 
of a wider behavioural syndrome involving aspects of the bold-shy personality continuum. We only investigated innovative behaviours as defined above, however, and not the social transmission of the behaviour through a population. The guppy is an ideal study organism in this regard, because innovation rate has already been well-studied (Laland and Reader, 1999a, 1999b; Reader \& Laland, 2000), which makes it easier to link innovation to any wider bold-shy behavioural syndrome of the type previously detected in guppies (Smith \& Blumstein, 2010), and other small fish species in aquarium studies (e.g., Dingemanse et al., 2007). Individuals with higher activity levels were predicted to explore faster and to be more innovative in novel tasks than less active individuals. Irrespective of activity levels, we also predict that bolder individuals showing more neophilic behaviours in novel environments and situations will tend to show higher rates of innovation. We also tested whether sex, body size and population of origin had an effect on the expression of these different behaviours. In a study by Laland and Reader (1999b), it was shown that female guppies were significantly more innovative than males. The authors suggested that this might be due to skewed parental investment, which is exclusively female in guppies, and this is supported by evidence that size has a greater effect on fecundity in females than in males (Laland \& Van Bergen, 2003). Females in our study were therefore expected to be more exploratory and innovative than the males. Laland and Reader (1999a) also showed that state-dependence can affect innovative behaviour in guppies, because individuals with higher hunger levels were more innovative than individuals with a lower hunger level. In guppies, like most fish with indeterminate growth, body size correlates significantly with age, and during scramble competition younger, smaller individuals are expected to be less competitive. Indeed, latency before completion of a novel foraging task has been shown to be shorter for younger compared to older male guppies (Laland \& Reader, 1999b). Smaller, younger individuals should therefore be more exploratory and innovative in general compared to larger individuals, as their lower competitive ability should increase their hunger and motivation for searching for new food sources (Laland \& Reader, 1999a).

\section{2 | METHODS}

\section{1 | Study species}

Guppies are small freshwater fish found in rivers and lakes on tropical islands in the general area of the Caribbean. The guppies used in this experiment were laboratory-reared at NTNU Norway, but originated from wild stocks from three rivers in Trinidad: Paria $\left(10^{\circ} 47^{\prime} \mathrm{N}\right.$ $\left.61^{\circ} 15^{\prime} \mathrm{W}\right)$; Campo $\left(10^{\circ} 41^{\prime} \mathrm{N}, 61^{\circ} 13^{\prime} \mathrm{W}\right)$; and Quare $\left(10^{\circ} 39^{\prime} \mathrm{N}\right.$ $61^{\circ} 12^{\prime} \mathrm{W}$ ). Approximately 500 individuals were collected from each river, and the three populations have been kept separated in six large aquaria (two per population, 400-500 L), approved by the Norwegian Animal Research Authority (licence number 7). The Paria population was collected in 1991, while the Campo and Quare populations were collected in 1998. Predation pressure was low in both the Paria and
Campo rivers, but high in the Quare River (Endler \& Houde, 1995; Pélabon et al., 2013). The laboratory populations have not been exposed to predators during the last 20 years, and any selection due to the regular captive environment is expected to be the same for all three populations. Among-population differences are therefore considered to have a historical genetic basis. The guppies were maintained on a 09:00-21:00 light-dark cycle at $25^{\circ} \mathrm{C}$ and were fed daily with dry flakes and freshly hatched brine shrimps (Artemia nauplii).

\section{2 | Experimental set-up}

In this study, each individual guppy was run through four behavioural tests: (a) exploration of a novel arena, (b) three-dimensional maze solving, and introduction to both (c) a novel object and (d) a novel food. Activity level was measured in each test. Most innovative behaviours in animals involve consummation of new food items, or inventing a new way to better process already known food items (Reader \& Laland, 2003b). It therefore seemed appropriate to include a novel food (d) as one of our innovation tests. In addition, in (b) we wanted to mimic the innovative assays involving a maze carried out by Laland and Reader (1999a), as these behaviours have already shown clear differences between the sexes. Exploration, activity and boldness to novel arenas and objects are behaviours traditionally used in the animal personality literature (e.g., Dingemanse et al., 2007), and (a) and (c) were therefore chosen as such standard tests that would allow us to link them with any individual differences in innovative behaviours. The dataset contained 19 males and 19 females from each of the three populations, adding up to a total of 114 individuals.

One male and one female guppy were randomly selected from each of the three populations and placed together in small isolation tanks $(22 \times 14.5 \times 14 \mathrm{~cm})$ in the same room as the experiment was conducted-one for each population-where they were food deprived for seven days prior to the experiment. Laland and Reader (1999b) showed that hunger level affected the innovation rate of male guppies, where hungrier individuals were more innovative (i.e., moved faster through a maze apparatus). By properly food depriving the guppies, hunger level was effectively standardized across all individuals, controlling out any differences in behaviour caused by contrasting hunger levels among individuals. We also hoped that this would increase motivation for approaching and eating the novel food, as well as activity in general by increasing the need to search for food. There were no deaths during or after the experiment, indicating that the food deprivation did not harm the guppies during the course of the experiment. Guppies of different body sizes were selected to ensure enough variation to estimate any effect of body size on the different behaviours. As there is a strong correlation between body size and age in guppies (Reznick, Butler, Rodd, \& Ross, 1996), we have included a wide age range in our subjects and some of the guppies included here might even have been considered juveniles.

Male and female guppies from each population were randomly assigned to one of six experimental tanks (see Figure 1), where individuals were run separately through each of the four behavioural assays. Above each tank there was a mounted camera that recorded 


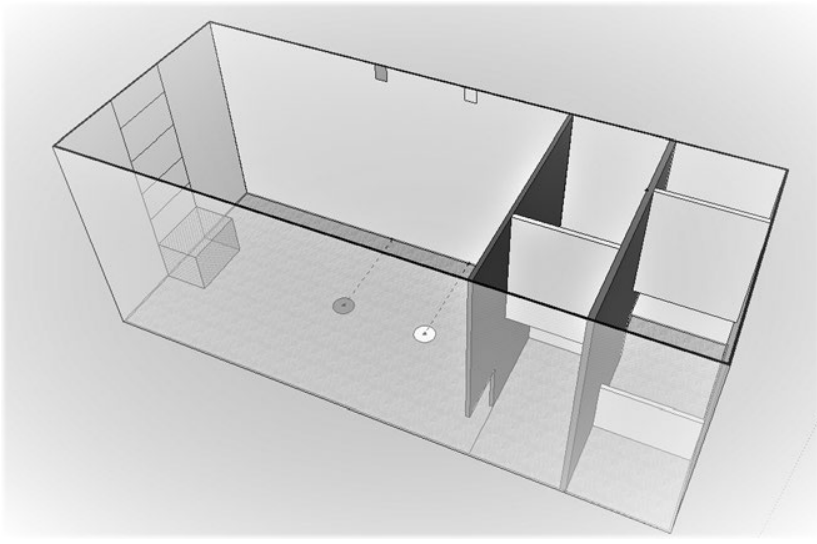

FIGURE 1 Tank set-up without the black plastic covering the walls. The tank was $61 \times 25 \times 24.5 \mathrm{~cm}$, and water was filled up to $18 \mathrm{~cm}$, similar to the tank size used by Laland and Reader (1999a; 1999b). The maze (on the right-hand side of the tank) consisted of two partitions that ran across the width of the tank $10 \mathrm{~cm}$ apart, with small square holes $(6 \times 6 \mathrm{~cm})$ at the bottom at opposite corners. Between these two partitions and between the inner partition and the glass wall, four additional partitions were fitted. The first and the third additional partitions were mounted $4 \mathrm{~cm}$ above the ground, while the second and fourth were $5 \mathrm{~cm}$ and $8 \mathrm{~cm}$ from the ground up, respectively, creating a 3-dimensional maze. In the novel arena (on the left-hand side of the tank), the white spot indicates the position of the novel object, while the grey spot indicates the position for the novel food. These locations were indicated during the trials by dark grey and light grey tape on the top of the glass wall. The box to the far left indicates the acclimation cage-see the text for more details. The maze door, not included in this figure, consisted of a plate placed over the hole in the first partition of the maze shown at the front of the tank (see Supporting Information Figure S1 for a view from above)

the movements of the fish. The walls of the tanks were covered in black plastic to keep individuals visually isolated from each other. Individuals were first placed in acclimation cages at one end of the tank where they were allowed ten minutes to settle prior to the start of the trial (Figure 1). After the acclimation period, the fish was gently encouraged to leave the cage, and the door was closed behind them to prevent re-entry. This was done to ensure that all guppies had the same amount of time to explore the tank and thus avoid any issues arising from individual differences in acclimation time versus exploration time among fish. The guppies were (a) given $30 \mathrm{~min}$ to explore the open area before the maze door was raised, and they were gently encouraged to enter the maze using a small landing net drawn slowly through the water. The fish did not seem to be affected by this procedure, as their swimming speed did not increase markedly at the time or immediately following. Again, this procedure was carried out to avoid any individually determined differences in the time available to different fish to explore the maze. The maze door was then closed, and the guppies had (b) $30 \mathrm{~min}$ to get to the end of the 3-dimentional maze. There was no food reward or hiding place at the end of the maze, hence the maze only represented innovation in terms of moving in a novel way through a novel environmental set-up (Laland \& Reader, 1999a). After the $30 \mathrm{~min}$ in the maze, the guppies were removed from the maze with a landing net and transferred back into the main exploration area where they were (c) presented with a novel object (an aquarium decoration), which had been placed in the middle of the exploration area; $10 \mathrm{~cm}$ from the maze wall (see Figure 1). After $15 \mathrm{~min}$, the novel object was removed, and the guppies were presented with (d) salmon food in a glass bowl, which was a novel food for these populations. The glass bowl had been placed in the tank before the start of the trials and was therefore part of the novel environmental in (a) and thus should not have represented a novel object in itself during (d). The novel food was placed in the middle of the tank, $10 \mathrm{~cm}$ from where the novel object had been, as indicated in Figure 1.

We measured body size after the guppies had completed all four behavioural trials. The guppies were sedated in ice water at $8^{\circ} \mathrm{C}$ and photographed individually with a $1 \mathrm{~cm}$ ruler next to them, before being put back into their isolation tanks where they recovered immediately. Body length was measured on the digitized image using tpsDIG, where landmarks were placed on the tip of the nose and the back of the tail (tail fin not included) and the distance assessed relative to landmarks placed on the ruler.

37 guppies ate the salmon food, meaning that it was perceived as a possible food source. The (a) exploration of the new area and (c) of the novel object can here be seen as traditional personality tests of exploration and boldness, respectively (Sih, Bell, \& Johnson, 2004), while (b) the maze and (d) the novel food represents innovative behaviours (Laland \& Reader, 1999a). Alternatively, the (a) exploration test and (b) the maze can be seen as spatial tests, while approach of the (c) novel object and (d) novel food can be seen as nonspatial tests. Guppies from different predatory regimes have been shown to differ in their exploratory strategies (Burns \& Rodd, 2008), with guppies living in high predator environments using more time to decide which chambers to explore in a maze, while guppies in low predatory environment made faster but less accurate decisions. Behaviours used in exploring an open field versus a maze might therefore be more strongly correlated with each other than with behaviours used in the approach of a novel object or a novel food item.

\subsection{1 | Video analysis}

All experiments were recorded using Sony high-resolution colour CCD cameras (model NC1381W) mounted above each of the six experimental tanks. Cameras were connected to H-264 Portable Mini Video Surveillance Recorders from LUPUS TEC (Recording resolution and frame rate: $704 \times 576 @ 25$ FPS, $352 \times 280 @ 25$ FPS (PAL)) on a $16 \mathrm{MbScanDisk}$ memory card.

Data recording started within $30 \mathrm{~s}$ of fish release from the acclimation cage, at the point when the surface water was sufficiently still to allow observations. To quantify the recorded data of (a) the exploration of a new area and (b) the maze, twenty-four squares were drawn on a transparent sheet and placed over the monitor screen for both trials (see Supporting Information Figure S1). Most individuals explored all 24 squares, making results on the number of new squares explored highly skewed to the maximum possible number. However, because not 
all individuals completed the assays, time to completion would also not fully represent all of the individual variation measured in these assays, because it would not differentiate between individuals that did and did not explore all of the squares. This problem of partial completion of the task was also the case for the other three assays, and the count and timing measurements for the different assays were therefore converted into rates (squares per time) prior to statistical analysis in order to capture all aspects of individual variation in behaviour. Exploration was quantified as the time an individual used to explore the total number of new squares divided by the total number of new squares explored. This created a rate representing the time used to enter/explore each square, where a low value indicates fast exploration. Speed through the maze was assessed by measuring the maximum distance reached by counting number of steps (12 steps in total with 2 squares per step; Supporting Information Figure S1) each guppy moved through the maze. A ratio with time to reach the maximum point in the maze divided by maximum point (step) reached was created to represent a rate of movement through the maze. Investigation of both the novel object and the novel food was quantified by how close the guppies got to the item, and the time taken to reach this distance. This was done by drawing five concentric circles $1 \mathrm{~cm}$ apart on a new transparent sheet placed on the monitor around the novel object and novel food (Supporting Information Figure S1). If the guppies did not enter any of the circles, they were given a score of one, while if they crossed the first circle they were scored with a value of two, and so on to the innermost circle where they were scored a value of six. Again, a ratio with time to reach minimum distance divided by minimum distance reach was created to represent the rate of approach towards the novel object and the novel food. Activity was measured in all four trials (i-iv) as the total number of squares visited in the first, middle and last 5 min of each trial. In the novel object trial, it was measured for the first, middle and last $3 \mathrm{~min}$, and the data were scaled up to equivalent values for 5 min for comparison in the other activity measures. Also, the smaller square sizes in the maze (see Supporting Information Figure S1) required further correction for activity during the maze trial to allow comparison with spatial activity in the main tank area. In this way, the activity level in terms of distance travelled per time period was equalized across the different types of assays.

\section{3 | Statistical analysis}

All the statistical analyses were carried out in $\mathrm{R}$, version $3.1 .1(\mathrm{R}$ Development Core Team, 2015). Activity measures were squareroot transformed prior to all statistical analyses to satisfy the assumption of normality.

Univariate ANCOVAs were carried out separately on all the eight behavioural variables (i.e., rate of exploration (a), speed through the 3dimentional maze (b), approach of both novel object (c) and novel food (d), and on activity measures for all four trials) to see if there were any mean differences between the three populations, sexes or any effect of body sizes, or their interactions. Full models with all interactions are presented in all cases. Experimenter identity (which of the two experimenters conducted the experiment), observer identity (which of the two experimenters analysed the videos) and tank identity were added as fixed effects to all the models, but subsequently removed from all results presented as they were shown to have no systematic effects. Mixed effect models were also used to calculate short-term repeatability for activity, as well as to investigate the habituation in activity level across the trials (see Supporting information).

Bivariate correlations between the eight different behavioural variables (i.e., the four rates and the four activity measures) were calculated using Pearson's correlation coefficients. This correlation matrix was produced for the overall results and for the two sexes separately (see Supporting Information Tables S5, S7 and S8). To then study the wider structure of these covariances between the different behaviour variables, structural equation modelling (SEM) was carried out using the correlation matrix in the lavaan package in R. Seven hypothesized covariance structures were proposed with different underlying latent variables (Figure 2 and Figure 3).

After running the first four hypotheses (Figure 2), we found no difference in ability to explain the data, suggesting that none of these models $(\mathrm{H} 1-\mathrm{H} 3)$ really captured the actual patterns of covariance. For example, we know that there was a high across-trial individual repeatability (Supporting Information Table S1) and strong correlations between all the activity variables (Supporting Information Table S5), and that the rate variables seemed correlated (in the nonspatial latent variable in $\mathrm{H} 3$ ). Therefore, exploratory principal component analyses (PCAs) were conducted to investigate possible syndrome structures not yet considered. The PCAs were run without a rotation and with a varimax rotation. A standard PCA adds principal components $(P C)$ where there is most variation left orthogonal to the previous $\mathrm{PC}$, while a varimax rotation rotates all PCs to find the structure that explains the most variation. Both versions of the PCAs were therefore run to better investigate the covariation structure of the data. From these results, three new a posteri hypothetical models were constructed and tested in SEM, as presented in Figure 3.

Structural equation modelling results were ranked according to the Akaike information criterion (AIC), and the best model was used to further explore any structural differences caused by the different sexes and populations, and to see if there was any influence of body size. The best model was run separately for each sex and population, with both free parameters and with parameters constrained to the factor loadings of the opposite sex or the two other populations (Dingemanse, Dochtermann, \& Wright, 2010). The best model was also run with body size covarying or not with the latent variables to see if it was implicated in the behavioural syndrome. AIC values were again used to see which of these models provided the best fit.

\section{RESULTS}

\subsection{Univariate tests of the variances in each behavioural variable}

There were very few significant effects of population, sex or body size on the average levels of each of the eight behavioural response variables, especially given the number of tests (Table 1, Figure 4). 
(a)

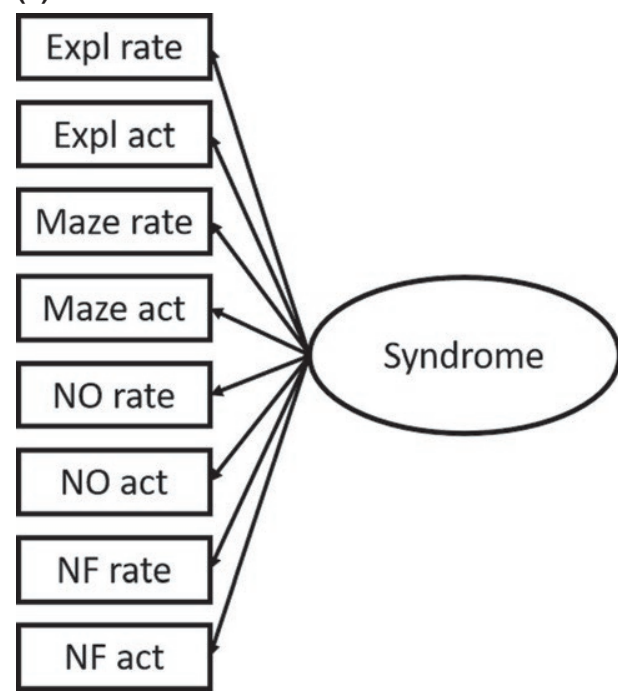

(b)

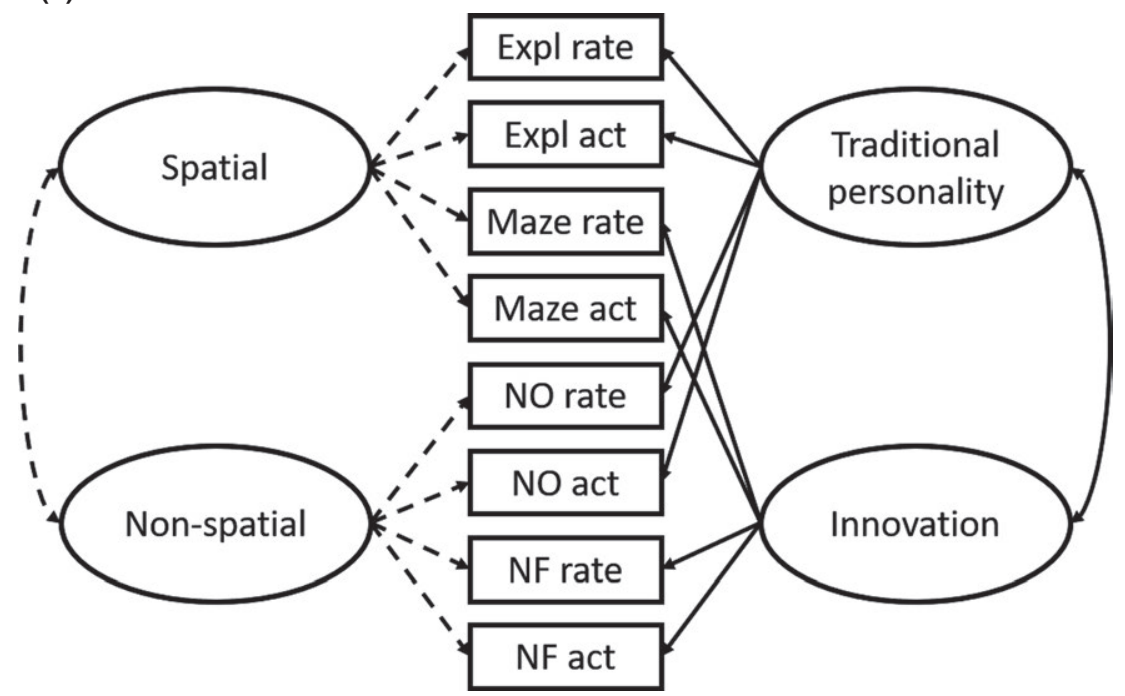

FIG URE 2 Causal diagrams of the four original hypotheses ( $\mathrm{HO}-\mathrm{H} 3$ ): (a) model $\mathrm{HO}$ (with no arrows active) where there is no covariance between any of the variables, and model $\mathrm{H} 1$ (arrows active) where all variables covary into one behavioural syndrome; and (b) model $\mathrm{H} 2$ (solid arrows active) where traditional personality behaviours and innovative behaviours constitute two separate latent variables, and model H3 (dashed arrows active) where spatial and nonspatial behaviours constitute two separate latent variables. Doubled-headed arrows between latent variables indicate covariance allowed between them. "act" indicates activity level measured in all trials, "Expl" indicates exploration in trial 1, while "NO" indicates novel object and "NF" novel food

(a)

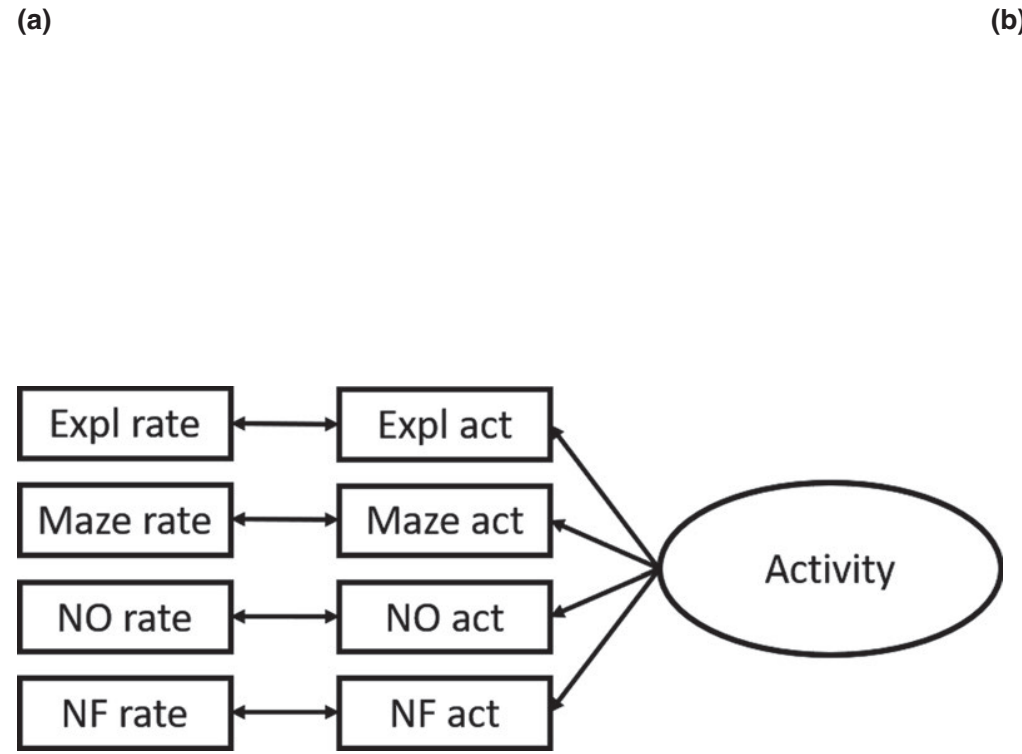

(b)

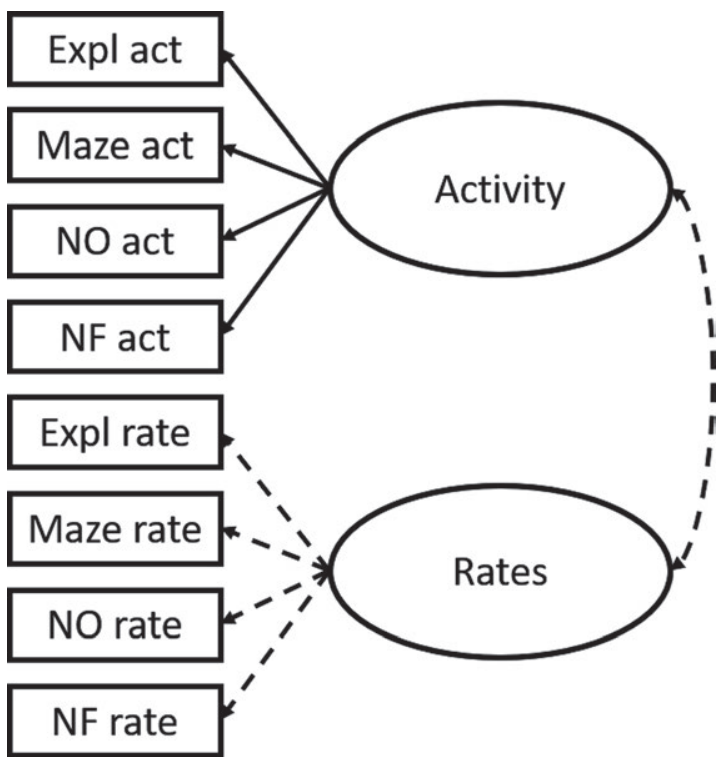

FIGURE 3 Causal diagrams for the three additional models inspired from the results of PCA (Tables 2 and 3): (a) model H4 where activity constitutes a latent variable, but activity correlates with the rate variable within the same test, hence activity within each trial drives the syndrome; and (b) model H5 (all arrows active; based on the un-rotated PCA) where activities and rates constitute two separate latent variables, and $\mathrm{H} 6$ (only solid arrows active; based on the varimax rotated PCA) with only activity as a latent variable, and the rates only added as variance terms. "act" indicates activity level measured in all trials, "Expl" indicates exploration in trial 1, while "NO" indicates novel object and "NF" novel food

In the novel food test, males did have a higher activity level than females, and smaller individuals were somewhat more active than larger ones, but the interaction term shows that this effect was largely restricted to females with their larger range of body sizes (Table 1; Supporting Information Figure S3). Population had no significant effect on any of the measured behaviours.
Activity level was highly individually repeatable across the assays in both sexes and all three populations, even with the inclusion of the maze trial (total $R=0.50$ (0.456-0.537); Supporting Information Table S1). However, this is repeatability across contexts and is only short-term repeatability, which might explain why it is perhaps higher than any true repeatability in activity assessed using 
TABLE 1 The effect of sex, population, body size and their interactions on the eight behaviour variables: (a) activity movement (_act) and (b) rates of exploration/innovation (_rate) in the four trials: exploration (expl_), maze (maze_), novel object (no_) and novel food (nf_). See text for details. Because of the number of tests, significant effects were set to $p<0.01$ and are shown in bold, while the standard significant effects $(p<0.05)$ are outlined by italics. The effect degrees of freedom are given for each parameter, and the error degrees of freedom was 102 in all models

\begin{tabular}{|c|c|c|c|c|c|c|c|c|c|}
\hline (a) Activities & $d f$ & \multicolumn{2}{|c|}{ expl_act } & \multicolumn{2}{|c|}{ maze_act } & \multicolumn{2}{|c|}{ no_act } & \multicolumn{2}{|c|}{ nf_act } \\
\hline Pop & 2 & 1.14 & 0.324 & 1.32 & 0.272 & 0.85 & 0.429 & 1.77 & 0.175 \\
\hline Sex & 1 & 4.90 & 0.029 & 3.74 & 0.056 & 0.73 & 0.395 & 7.42 & 0.008 \\
\hline Size & 1 & 5.02 & 0.027 & 3.53 & 0.063 & 3.55 & 0.062 & 8.05 & 0.005 \\
\hline Sex:size & 1 & 4.72 & 0.032 & 3.76 & 0.055 & 0.63 & 0.430 & 7.19 & 0.009 \\
\hline \multirow[t]{2}{*}{ Pop:sex:size } & 2 & 1.32 & 0.272 & 0.64 & 0.531 & 0.13 & 0.874 & 1.27 & 0.284 \\
\hline & & \multicolumn{2}{|c|}{ expl_rate } & \multicolumn{2}{|c|}{ maze_rate } & \multicolumn{2}{|c|}{ no_rate } & \multicolumn{2}{|c|}{ nf_rate } \\
\hline (b) Rates & $d f$ & $F$ & $p$ & $F$ & $p$ & $F$ & $p$ & $F$ & $p$ \\
\hline Size & 1 & 0.16 & 0.693 & 0.01 & 0.937 & 0.88 & 0.351 & 0.43 & 0.512 \\
\hline Pop:sex & 2 & 0.02 & 0.979 & 3.92 & 0.023 & 2.28 & 0.108 & 0.83 & 0.438 \\
\hline Pop:size & 2 & 1.71 & 0.187 & 0.24 & 0.788 & 0.47 & 0.626 & 1.56 & 0.215 \\
\hline Sex:size & 1 & 1.21 & 0.273 & 5.98 & 0.016 & 0.63 & 0.428 & 0.15 & 0.695 \\
\hline Pop:sex:size & 2 & 0.06 & 0.939 & 3.71 & 0.028 & 2.24 & 0.111 & 0.70 & 0.501 \\
\hline
\end{tabular}

the same assay or context over a sufficiently long period of different days or months (Bell, Hankison, \& Laskowski, 2009).

\subsection{1 | Nonrotated and varimax rotated PCAs}

PCAs were conducted to further investigate the covariance structure between the eight behaviour variables. These were done without a rotation (Table 2 ) and with a varimax rotation (Table 3 ). In the un-rotated PCA, all the activity variables in addition to rate during the novel food test dropped out in the first principal component (PC), while all the other rates dropped out in PC2. These were the only significant PC's (eigenvalue $>1$ ) with eigenvalue 3.33 and 1.38 , respectively. In the varimax rotated PCA, all the activity measurements dropped out as PC1, while the rates dropped out singularly as PC2-PC5. Only the first PC was significant though, with an eigenvalue of 1.97 .

\subsubsection{SEM of covariance patterns between the behavioural variables}

To investigate the structure of the behavioural syndrome, seven SEM models were compared (see Methods, Figures 2 and 3). The HO hypothesis, where none of the observed variables correlate, had the worst fit (see Table 4), which indicates that there is some sort of syndrome involving these variables. However, there were no meaningful differences between $\mathrm{H} 1$ (all behaviours correlate), $\mathrm{H} 2$ (personality vs. innovation) and $\mathrm{H} 3$ (spatial vs. nonspatial)-see Figure 2, which is why we included three new models based on the results from the PCA (see Methods). Of these, $\mathrm{H} 5$ (activity vs. rate) emerged as clearly the best model (Figure 5a), with $\mathrm{H} 4$ (activity driven) and $\mathrm{H} 6$ (only activity) actually having higher AIC scores than $\mathrm{H} 1-\mathrm{H} 3$ (Table 4). This suggests that the rates of exploration/innovation were not simply driven by the activity level during the different assays, but that the rate variable in themselves constitutes a separate set of covariances captured by the second latent variable, which then covaries with the latent variable containing the activity measures (Figure $5 \mathrm{a}$ ). The factor loadings for activity in $\mathrm{H} 5$ are higher than those for the rate variables, indicating that activity has a stronger effect within the syndrome, although $\mathrm{H} 5$ does allow us to conclude that there was a separate lesser covariance within the rate variables as well. The covariance between the latent variables is negative, as expected, as more active individuals were more likely to complete the tasks faster, such as explore the new area or get through the maze (note that lower rates refer to a faster completion of a trial). All loadings for the rate variables were positive, thus bolder and more exploratory individuals were also more innovative. There were no significant differences in the syndrome structure for this model between the sexes or between the three populations (see Supporting Information Tables S6-S8).

Including body size as part of the activity syndrome of the best model ( $\mathrm{H} 5$ ) gave the best fit (Table 5, Figure 5 b). However, because the $\triangle \mathrm{AIC}$ value is $<2$, it does not have better fit than models where body size is part of the rate latent variable or linked to both latent variables. However, it is better than $\mathrm{H} 5$ with only the variance term for body 
FIGURE 4 Mean \pm SE for the two sexes in all three populations for: (a) activity during the exploration trial (number of squares visited); (b) exploration rate (time to explore new squares divided by number of new squares visited); (c) exploration of the maze (time to get to maximum point divided by maximum point reached); (d) approach to the novel object (time to reach minimum distance to the novel object divided by minimum distance reached); and (e) approach to the novel food (time to reach minimum distance to the novel food divided by minimum distance reached). The activity measurements (the total number of squares visited in the first, middle and last $5 \mathrm{~min}$ ) for the maze, the novel object and the novel food trials were all qualitatively similar to the exploration trial results in (a) and are therefore not shown here for reasons of brevity. See Methods for more information on each measure
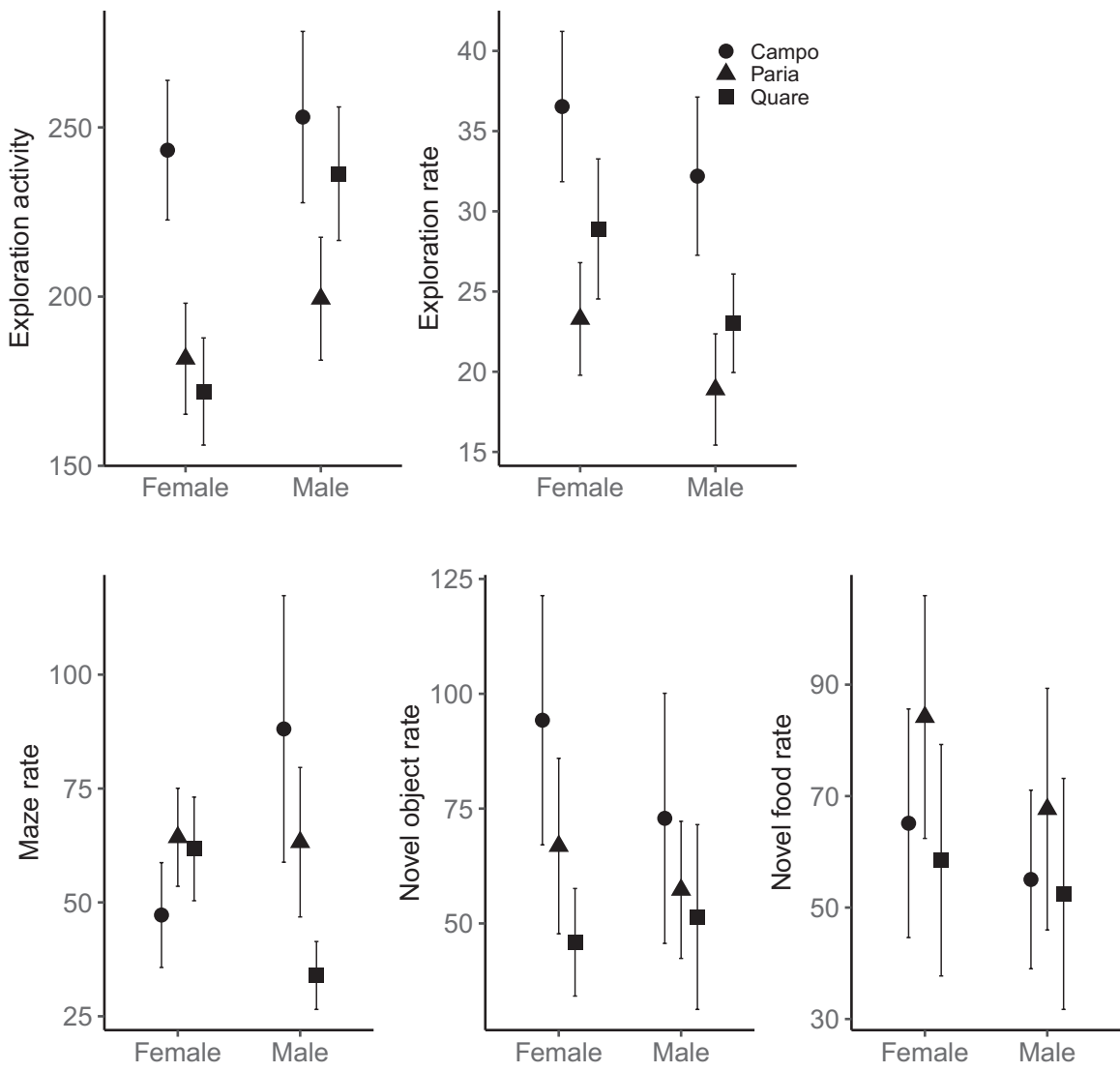

TAB LE 2 Results from the un-rotation PCA. Eigenvalues and the proportion of the variance explained are given for all the PCs, in addition to the loadings for each variable within these PCs. Strong loadings $(>0.30)$ are shown in bold

\begin{tabular}{|lccc|}
\hline & PC1 & PC2 & PC3 \\
\hline Eigenvalue & 3.33 & 1.38 & 0.96 \\
\hline $\begin{array}{l}\text { Proportion of } \\
\text { variance }\end{array}$ & 0.42 & 0.17 & 0.12 \\
\hline $\begin{array}{l}\text { Cumulative variance } \\
\text { expl_act }\end{array}$ & 0.42 & 0.59 & 0.71 \\
\hline expl_rate & 0.84 & 0.05 & 0.05 \\
\hline maze_act & -0.18 & 0.58 & 0.61 \\
\hline maze_rate & 0.83 & 0.18 & 0.24 \\
\hline no_act & -0.14 & -0.71 & -0.38 \\
\hline no_rate & 0.89 & 0.15 & -0.02 \\
\hline nf_act & -0.18 & 0.65 & -0.37 \\
\hline nf_rate & 0.91 & 0.12 & 0.01 \\
\hline
\end{tabular}

size included in the model $(\triangle \mathrm{AIC}>2$ ), indicating that body size covaried with the behavioural syndrome structure in the guppies in some way. Body size had a negative factor loading (see Figure $5 \mathrm{~b}$ ), which confirms the effects seen above (Table 1, Supporting Information Figure S3). Smaller individuals were more active than larger individuals, suggesting that the behavioural syndrome identified here may be condition-dependent in terms of differences in the level of individual indeterminate growth and/or development.
TABLE 3 Results from the PCA with a varimax rotation. Eigenvalues and the proportion of the variance explained are given for all the PCs, in addition to the loadings for each variable within these PCs. Strong loadings $(>0.30)$ are shown in bold

\begin{tabular}{|cccccc} 
& PC1 & PC2 & PC3 & PC4 & PC5 \\
\hline $\begin{array}{l}\text { Eigenvalue } \\
\begin{array}{l}\text { Proportion of } \\
\text { variance }\end{array}\end{array}$ & 1.97 & 1.03 & 1.01 & 1.01 & 1.00 \\
$\begin{array}{l}\text { Cumulative } \\
\text { variance }\end{array}$ & 0.25 & 0.13 & 0.13 & 0.13 & 0.13 \\
$\begin{array}{l}\text { expl_act } \\
\text { expl_rate }\end{array}$ & -0.42 & -0.15 & -0.04 & -0.04 & -0.05 \\
maze_act & 0.49 & -0.07 & -0.09 & 0.04 & 0.01 \\
maze_rate & 0.01 & 0.03 & 0.98 & 0.09 & 0.13 \\
no_act & 0.91 & -0.16 & 0.05 & -0.05 & -0.06 \\
\hline no_rate & -0.05 & 0.05 & 0.13 & 0.05 & 0.99 \\
nf_act & 0.83 & -0.15 & -0.03 & -0.06 & -0.02 \\
\hline nf_rate & -0.17 & 0.97 & 0.03 & 0.09 & 0.05 \\
\hline
\end{tabular}

\section{4 | DISCUSSION}

In this study, we show that innovative behaviours covary with traditional personality behaviours as part of a wider behavioural syndrome in guppies. As the best model including activity and rates as separate latent variables $(\mathrm{H} 5)$ had a better fit than a model with covariances in only activity $(\mathrm{H} 6)$, it is clear that covariation between the 
TAB LE 4 Comparison of the seven SEM models (see Figures 2 and 3) using AIC values, where $\mathrm{K}$ is the number of parameters estimated. The $\mathrm{H} 5$ (activity vs. rates) has a better fit than the other models $(\triangle \mathrm{AIC}>2)$

\begin{tabular}{|llllr|}
\hline Model & Name & $K$ & AIC & \multicolumn{1}{c|}{$\Delta$ AIC } \\
\hline H5 & $\begin{array}{l}\text { Activity versus } \\
\text { rates }\end{array}$ & 17 & 2283.92 & 0.00 \\
\hline H3 & $\begin{array}{l}\text { Spatial versus } \\
\text { nonspatial }\end{array}$ & 17 & 2287.59 & 3.67 \\
\hline H1 & All correlates & 16 & 2287.68 & 3.76 \\
\hline H2 & $\begin{array}{l}\text { Personality versus } \\
\text { innovation }\end{array}$ & 17 & 2289.59 & 5.67 \\
\hline H6 & Only activity & 12 & 2298.15 & 14.23 \\
\hline H4 & Activity driven & 16 & 2300.85 & 16.93 \\
\hline H0 & No correlations & 8 & 2596.11 & 312.19 \\
\hline
\end{tabular}

exploration/investigation rates also contributed to the syndrome, and that they were not merely driven by their covariance with levels of activity within the same test (i.e., model H4, see Figure 3). The innovative behaviours (maze solving and exploitation of novel food) and classical behaviours measured in studies of animal personality (exploration and boldness towards a novel object) did not divide into separate latent variables (model $\mathrm{H} 2$, see Figure 2). It therefore seems that the classification of the innovation versus classical animal personality behaviours in this way might be an artificial construct arising from the different research traditions (e.g., see the different books: Reader \& Laland, 2003a, Carere \& Maestripieri, 2013). Approaching a novel object and novel food might not be any different in any fundamental way for the guppies, even though they could be categorized by different groups of researchers as boldness versus innovation behaviours, respectively. Thus, the innovative
TABLE 5 Further SEM comparisons using just the best model (H5-activity vs. rate-see Figure 5) was performed to see if body size had any effect on the syndrome structure, using AIC values, where $K$ is the number of parameters estimated. Body size was included in the best model ( $\mathrm{H} 5$-activity vs. rate) as a variance term with either no covariance with any of the latent variables or as part of either the activity syndrome or the rate syndrome, or both. The model with the best fit is the one where body size is included as part of the "activity syndrome" (see Figure 5b), and this model is better than the "no body size syndrome" model where only the variance term for body size is included. It is, however, not better than when body size is added as part of the "rate syndrome" or "both syndromes."

\begin{tabular}{|llll|}
\hline Model & K & AIC & AAIC \\
\hline Activity syndrome & 19 & 2606.22 & 0.00 \\
\hline Rate syndrome & 19 & 2607.00 & 0.78 \\
\hline Both syndromes & 20 & 2608.00 & 1.78 \\
\hline $\begin{array}{l}\text { No body size } \\
\text { syndrome }\end{array}$ & 18 & 2608.43 & 2.21 \\
\hline
\end{tabular}

behaviours we measured (i.e., the maze and novel food trials) might not necessarily have been "innovation" behaviours at all and might just reflect differences in boldness and shyness in the exploration of novelty. However, these assays were specifically chosen because they are similar to tests that have previously been used to measure innovation for guppies in studies by Laland and Reader (1999a). As previously mentioned, the utilization of a novel food is the most common form of innovative behaviour and has become a focus of many studies in this area (Reader \& Laland, 2003a). As the exploration/investigation rate variables here constitute a single latent variable, and all the loadings for the rates where positive, this suggests that this variation could be driven by individual differences in (a)

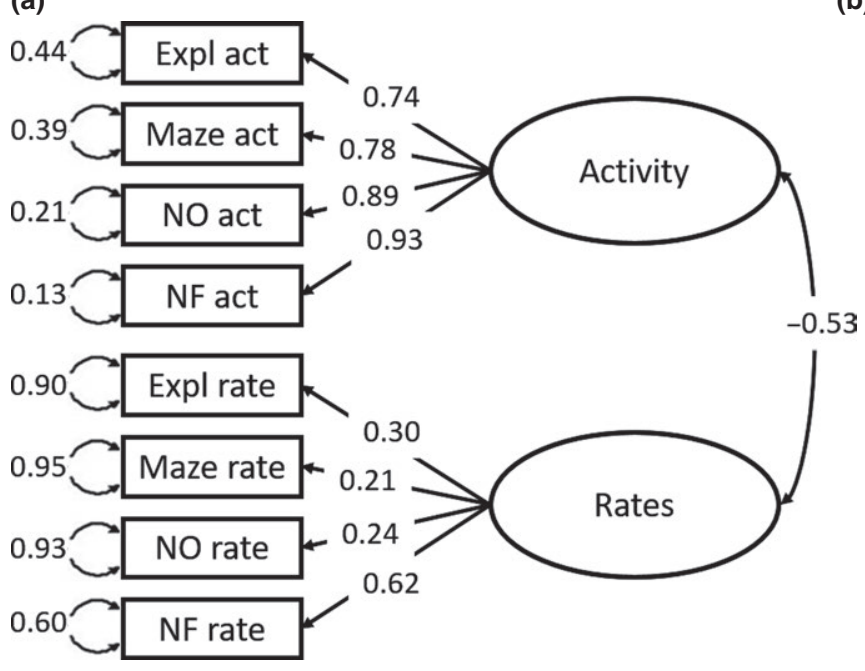

(b)

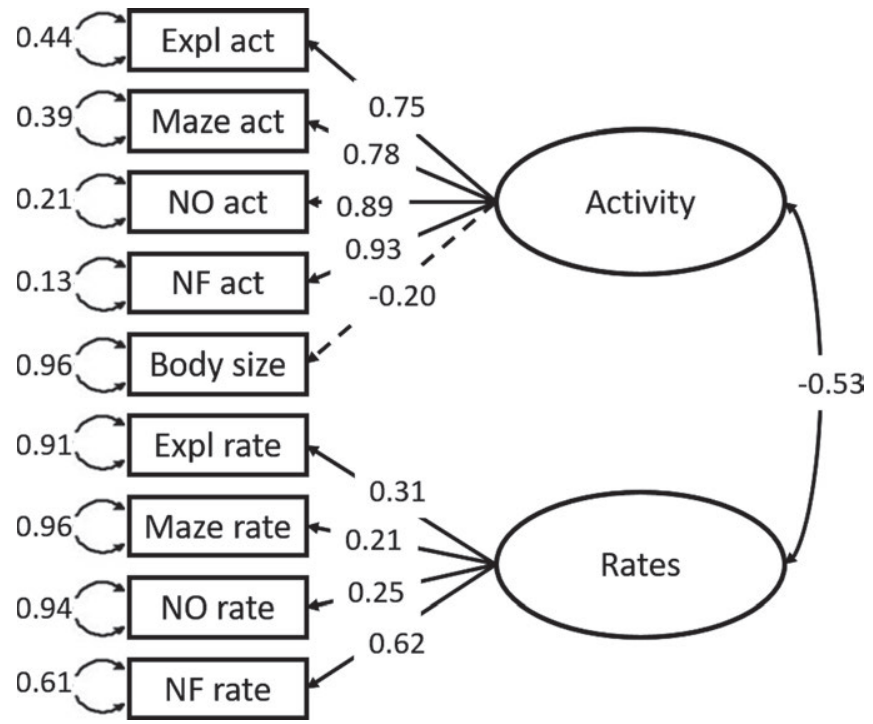

FIGURE 5 The best model (H5-activity vs. rate) with factor loadings (one-headed arrows) and error variance of the observed variables (squares) that is left unexplained by the latent variable (circles). The double-headed arrow between the latent variables shows the correlation between them: (a) without body size (as in Table 4); and (b) body size included in the latent variable for activity (as in Table 5). Negative factor loadings for body size shows that smaller individuals were more active than larger individuals 
curiosity, neophilia/neophobia, boldness and hence a tendency to innovate. This therefore suggests that individuals that explore faster are also in general more bold and innovative. The negative correlation between the latent variables for activity and rates also indicates that more active individuals finished the different tasks quicker than less active individuals (as mentioned above, a lower rate score refers to fast completion of the tasks), showing that bolder and more innovative individuals were also more active.

Population and sex had no effect on the behavioural syndrome structure. These guppies have been in captivity for over 20 years, and they have not been under any specific selection pressure, such as predation or food limitation, during this time. Dingemanse et al. (2007) showed that three-spine sticklebacks living in predator-free environments had weaker syndrome structures compared with sticklebacks that lived in areas with predators. Predation pressure is the main difference between our guppy populations in their original sites, where predator fish were absent in Paria and Campo, whereas Quare experienced high predation risk (Pélabon et al., 2013; Reznick et al., 1996), and behavioural differences between wild guppy populations due to difference in predation pressure have been shown previously (e.g., Burns \& Rodd, 2008, Harris, Ramnarine, Smith, \& Pettersson, 2010). The lack of any predator pressure on all the three populations over many generations in captivity might therefore have reduced such behavioural differences among these populations. In other animals, captive strains can exhibit substantial changes in behaviour after only a few generations (Bolstad et al., 2012; Larsen et al., 2011; Smith \& Blumstein, 2008; Williams \& Hoffman, 2009). It is therefore problematic to come to any firm conclusions regarding our comparisons between the decedents of these three natural populations.

From the univariate analysis, no effect of sex was found on the exploration/investigation rate variables, including anything that could be described as innovation. However, there were some differences between the sexes in activity levels, which interestingly involved larger females being less active than males (that showed no effect of body size over their smaller range of body sizes; Supporting Information Figure S3), and the effect was especially clear in the context of a novel food. Female fitness depends more in longevity since larger, and thus older, females produce more offspring. In addition, females can store sperm and do not necessarily need to seek out males to produce more broods (Reznick \& Yang, 1993). Males, on the other hand, constantly need to seek out new mates to increase their reproductive outcome as females are the choosy sex, and thus perhaps males have to take more risks (Houde, 1988). Female guppies caught in the wild show more cautious behaviour when emerging from a shelter, as compared to males (Harris et al., 2010). In our study, the guppies were not allowed to emerge from the shelter by their own accord, but the lower activity level in females might reflect their more cautious nature in this new environment. In addition, female guppies tend to spend more time in shoals than males (Magurran, Seghers, Carvalho, \& Shaw, 1992), and the females in our study might therefore have been more affected by the separation from the rest of the shoal than the males were, resulting in their relatively lower activity levels. Boldness has been shown to correlate with sociality in female guppies (Trompf \& Brown, 2014), and this might explain our results in terms of differences we find in solitary assays of activity and exploration between the sexes. However, these arguments are hard to reconcile with published work on innovation in this species (Laland and Reader, 1999a, 1999b; Laland \& Van Bergen, 2003). Namely, we found none of the predicted sex differences in innovation found in these previous studies. One reason for the contrasting results here versus accounts in the published literature concerning both population and sex differences in guppy behaviour could be due to the different origins and holding conditions of the fish used in the different laboratories. It is therefore hard to interpret these sorts of results from different aquarium studies of different populations that have been domesticated for varying periods of time, with or without more or less natural selective forces. This has been shown in another model species in behavioural studies, the zebra finch (Taeniopygia guttata), where domesticated populations were found to differ significantly from the wild population in many important aspects of their behaviour, and also from each other (Forstmeier, Segelbacher, Mueller, $\&$ Kempenaers, 2007). Clearly, more standardized comparisons of this type are needed, and behavioural studies like this should preferably be carried out on exclusively recently wild-caught populations or captive populations of known selective differences to be able to draw any ecological or evolutionary conclusions.

Body size was part of the syndrome structure, where smaller individuals were more active than larger individuals. Among-individual differences in body size might therefore have been driving the whole behavioural syndrome in this study system. The univariate tests also reflected this effect of body size on activity, but mostly in females with their larger range of body sizes. Males were significantly more active than females, and not simply due to their smaller size compared with females. All of these effects were most obvious in the novel food trial, which was the only trial that involved food being present, clearly suggesting a foraging context for these differences. Body size is expected to be an important factor for female fecundity (Reznick \& Yang, 1993), which might cause smaller females to search more for food and thus be more active than both males and larger females. Because body size was fixed over the timescale of the experiment and highly correlated with age in guppies (Reznick et al., 1996), activity level might be driven by the relative developmental state of the individual. Smaller fish might need to search more for new sources of food, either due to more energy needed for growth or lower competitive ability around already discovered foods. To show that the behaviours here reflect animal personalities, repeated measures must be taken at different developmental states, to show individual behavioural consistency over a longer time period independent of developmental state. In this way, it might also be possible to examine whether differences in individual growth rates affect the syndrome structure we identified here. Individuals with a more rapid growth might be expected to take more risks in the form of exploration and innovation, as part of a "pace-of-life" syndrome where fast-growing individuals live fast and die young (Réale, Garant, et al., 2010). 


\section{5 | CONCLUSIONS}

For our guppy populations, the behavioural syndrome structure was a mixture of personality and innovation behaviours, with no biological basis for this dichotomy in behaviours apart from their respective research traditions. Classifying behaviours as innovation and animal personalities might therefore be arbitrary, and reconciling these two fields might be beneficial for a better understanding of how different aspects of animal behaviour is integrated.

The lack of any effects of both sex and population in behaviours, where they have been shown to differ in earlier studies, indicates the difficulty in obtaining consistent results when comparing traits between different domesticated populations. In addition, most of the natural selection pressures that might have been responsible for any patterns in these behaviours might have been weakened or removed entirely from such long-term domesticated populations. It is therefore challenging to find firm answers to predictions based on the ecology of the original wild habitat for such long-term captive populations. We suggest that future experiments of this type should be conducted on wild-caught populations where the natural selection pressures are known, and then tested in a laboratorial set-up where it is possible to control for any confounding (e.g., social) effects on behavioural variation within and between individuals.

Body size appeared to be part of the behavioural syndrome, and we therefore suggest that differences in individual developmental state might actually drive the whole syndrome documented here. To get a better understanding of how developmental state affects activity levels, repeated behavioural measures would be required for the same individuals in different developmental states. This could also be used to see if there is any particular developmental state when innovative behaviours are more important in creating individual differences, either because more food resources are needed at that point due to rapid growth (or reproduction), or just because smaller, younger individuals have lower competitive abilities and need to innovate and find their own food resources. By following individuals over a longer period of time, it might also be possible to record individual differences due to contrasting early life experiences. For instance, how food availability during early development affects mean levels of behaviour and wider syndrome structures, and this could confirm if our results here are part of a wider pace-of-life syndrome (sensu Réale, Garant, et al., 2010).

In conclusion, behaviours across different areas of study, such as innovation and cognitive problem-solving, should be added to the animal personality research to get a better picture of how behaviours across a wider range of adaptive contexts are integrated. In addition, such studies of wider behavioural syndromes should be carried out on wild populations under natural selection, as well as across different developmental timeframes to get a better understanding of the origins behind the structure of behavioural syndromes.

\section{ACKNOWLEDGEMENTS}

We thank Anne-Lise Haugdahl Humstad and Henriette Vaageland for helping out with the experimental set-up and data collection. We would also like to thank Øystein Hjorthol Opedal and Endre Grüner Ofstad for their help with the statistical analysis, and Ned Dochtermann and Niels Dingemanse for helpful comments on an earlier version of this work. This work was partly supported by the Research Council of Norway through its Centres of Excellence funding scheme, project number 223257.

\section{ORCID}

Monica Anderson Berdal (iD http://orcid. org/0000-0003-1573-2370

\section{REFERENCES}

Bell, A. M., Hankison, S. J., \& Laskowski, K. L. (2009). The repeatability of behaviour: a meta-analysis. Animal Behaviour, 77, 771-783. https:// doi.org/10.1016/j.anbehav.2008.12.022

Benus, R. F., Koolhaas, J. M., \& Van Oortmerssen, G. A. (1988). Aggression and adaptation to the light-dark cycle: Role of intrinsic and extrinsic control. Physiology \& Behavior, 43, 131-137. https://doi. org/10.1016/0031-9384(88)90228-4

Bolstad, G. H., Pelabon, C., Larsen, L. K., Fleming, I. A., Viken, Å., \& Rosenqvist, G. (2012). The effect of purging on sexually selected traits through antagonistic pleiotropy with survival. Ecology and Evolution, 2, 1181-1194. https://doi.org/10.1002/ece3.246

Burns, J. G., \& Rodd, F. H. (2008). Hastiness, brain size and predation regime affect the performance of wild guppies in a spatial memory task. Animal Behaviour, 76, 911-922. https://doi.org/10.1016/j. anbehav.2008.02.017

Carere, C., \& Maestripieri, D. (2013). Animal personalities: behavior, physiology, and evolution. Oxford, UK: University of Chicago Press.

Dingemanse, N. J., Dochtermann, N. A., \& Wright, J. (2010). A method for exploring the structure of behavioural syndromes to allow formal comparison within and between data sets. Animal Behaviour, 79, 439-450. https://doi.org/10.1016/j.anbehav.2009.11.024

Dingemanse, N. J., Wright, J., Kazem, A. J. N., Thomas, D. K., Hickling, R., \& Dawnay, N. (2007). Behavioural syndromes differ predictably between 12 populations of three-spined stickleback. Journal of Animal Ecology, 76, 1128-1138. https://doi. org/10.1111/j.1365-2656.2007.01284.x

Endler, J. A., \& Houde, A. E. (1995). Geographic variation in female preferences for male traits in Poecilia reticulata. Evolution, 49, 456-468.

Fisher, J., \& Hinde, R. A. (1949). The opening of milk bottles by birds. British Birds, 42, 347-357.

Forstmeier, W., Segelbacher, G., Mueller, J. C., \& Kempenaers, B. (2007). Genetic variation and differentiation in captive and wild zebra finches (Taeniopygia guttata). Molecular Ecology, 16, 4039-4050.

Goodall, J. (1964). Tool-using and aimed throwing in a community of free-living chimpanzees. Nature, 201, 1264. https://doi. org/10.1038/2011264a0

Griffin, A. S., Guez, D., Lermite, F., \& Patience, M. (2013). Tracking changing environments: innovators are fast, but not flexible learners. Plos One, 8, e84907.

Griffin, A. S., Guillette, L. M., \& Healy, S. D. (2015). Cognition and personality: an analysis of an emerging field. Trends in Ecology \& Evolution, 30, 207-214. https://doi.org/10.1016/j.tree.2015.01.012 
Guillette, L. M., Reddon, A. R., Hoeschele, M., \& Sturdy, C. B. (2011). Sometimes slower is better: slow-exploring birds are more sensitive to changes in a vocal discrimination task. Proceedings of the Royal Society of London B: Biological Sciences, 278, 767-773. https://doi. org/10.1098/rspb.2010.1669

Guillette, L. M., Reddon, A. R., Hurd, P. L., \& Sturdy, C. B. (2009). Exploration of a novel space is associated with individual differences in learning speed in black-capped chickadees, Poecile atricapillus. Behavioural Processes, 82, 265-270. https://doi.org/10.1016/j. beproc.2009.07.005

Harcourt, J. L., Biau, S., Johnstone, R., \& Manica, A. (2010). Boldness and information use in three-spined sticklebacks. Ethology, 116, 440447. https://doi.org/10.1111/j.1439-0310.2010.01757.x

Harris, S., Ramnarine, I. W., Smith, H. G., \& Pettersson, L. B. (2010). Picking personalities apart: estimating the influence of predation, sex and body size on boldness in the guppy Poecilia reticulata. Oikos, 119, 1711-1718. https://doi.org/10.1111/j.1600-0706.2010.18028.x

Hoppitt, W., \& Laland, K. N. (2013). Social learning: an introduction to mechanisms, methods, and models. Oxford, UK: Princeton University Press.

Hosey, G. R., Jacques, M., \& Pitts, A. (1997). Drinking from tails: social learning of a novel behaviour in a group of ring-tailed lemurs (Lemur catta). Primates, 38, 415-422. https://doi.org/10.1007/BF02381881

Houde, A. E. (1988). The effects of female choice and male-male competition on the mating success of male guppies. Animal Behaviour, 36, 888-896. https://doi.org/10.1016/S0003-3472(88)80171-4

Katzir, G. (1982). Relationships between social structure and response to novelty in captive jackdaws, Corvus monedula L. I. Response to Novel Space.Behaviour, 81, 231-263.

Laland, K. N., \& VanBergen, Y. (2003). Experimental studies of innovation in the guppy.

Laland, K. N., \& Reader, S. M. (1999a). Foraging innovation in the guppy. Animal Behaviour, 57, 331-340.

Laland, K. N., \& Reader, S. M. (1999b). Foraging innovation is inversely related to competitive ability in male but not in female guppies. Behavioral Ecology, 10, 270-274.

Larsen, L. K., Pelabon, C., Bolstad, G., Viken, Å., Fleming, I., \& Rosenqvist, G. (2011). Temporal change in inbreeding depression in life-history traits in captive populations of guppy (Poecilia reticulata): evidence for purging? Journal of Evolutionary Biology, 24, 823-834. https://doi. org/10.1111/j.1420-9101.2010.02224.x

Light, K. R., Kolata, S., Hale, G., Grossman, H., \& Matzel, L. D. (2008). Upregulation of exploratory tendencies does not enhance general learning abilities in juvenile or young-adult outbred mice. Neurobiology of Learning and Memory, 90, 317-329. https://doi.org/10.1016/j. nlm.2008.04.007

Liker, A., \& Barta, Z. (2002). The effects of dominance on social foraging tactic use in house sparrows. Behaviour, 139, 1061-1076. https://doi. org/10.1163/15685390260337903

Magurran, A., Seghers, B., Carvalho, G., \& Shaw, P. (1992). Behavioural consequences of an artificial introduction of guppies (Poecilia reticulata) in N. Trinidad: evidence for the evolution of anti-predator behaviour in the wild. Proceedings of the Royal Society of London B: Biological Sciences, 248, 117-122.

Mathot, K. J., Wright, J., Kempenaers, B., \& Dingemanse, N. (2012). Adaptive strategies for managing uncertainty may explain personality-related differences in behavioural plasticity. Oikos, 121, 10091020. https://doi.org/10.1111/j.1600-0706.2012.20339.x

Matzel, L. D., Townsend, D. A., Grossman, H., Han, Y. R., Hale, G., Zappulla, M., ... Kolata, S. (2006). Exploration in outbred mice covaries with general learning abilities irrespective of stress reactivity, emotionality, and physical attributes. Neurobiology of Learning and Memory, 86, 228-240. https://doi.org/10.1016/j.nlm.2006.03.004

Morand-Ferron, J., Cole, E. F., Rawles, J. E., \& Quinn, J. L. (2011). Who are the innovators? A field experiment with 2 passerine species.
Behavioral Ecology, 22, 1241-1248. https://doi.org/10.1093/beheco/ arr120

Niemela, P. T., Vainikka, A., Hedrick, A. V., \& Kortet, R. (2012). Integrating behaviour with life history: boldness of the field cricket, Gryllus integer, during ontogeny. Functional Ecology, 26, 450-456. https://doi. org/10.1111/j.1365-2435.2011.01939.x

Pélabon, C., Bolstad, G. H., Egset, C. K., Cheverud, J. M., Pavlicev, M., \& Rosenqvist, G. (2013). On the relationship between ontogenetic and static allometry. The American Naturalist, 181, 195-212. https://doi. org/10.1086/668820

Ramsey, G., Bastian, M. L., \& van Schaik, C. (2007). Animal innovation defined and operationalized. Behavioral and Brain Sciences, 30, 393407. https://doi.org/10.1017/S0140525X07002373

Reader, S. M., \& Laland, K. N. (2000). Diffusion of foraging innovations in the guppy. Animal Behaviour, 60, 175-180. https://doi.org/10.1006/ anbe. 2000.1450

Reader, S. M., \& Laland, K. N. (2003a). Animal innovation. Oxford, UK: Oxford University Press.

Reader, S. M., \& Laland, K. N. (2003b). Animal innovation: an introduction. Oxford, UK: Oxford University Press, Oxford.

Réale, D., Dingemanse, N. J., Kazem, A. J., \& Wright, J. (2010). Evolutionary and ecological approaches to the study of personality. Philosophical Transactions of the Royal Society of London. Series B, Biological Sciences, 365(1560), 3937-3946.

Réale, D., Garant, D., Humphries, M. M., Bergeron, P., Careau, V., \& Montiglio, P.-O. (2010). Personality and the emergence of the pace-of-life syndrome concept at the population level. Philosophical Transactions of the Royal Society of London B: Biological Sciences, 365, 4051-4063.

Reznick, D. N., Butler, M. J. IV, Rodd, F. H., \& Ross, P. (1996). Life-history evolution in guppies (Poecilia reticulata) 6. Differential mortality as a mechanism for natural selection. Evolution, 50(4), 1651-1660. https://doi.org/10.2307/2410901

Reznick, D., \& Yang, A. P. (1993). The influence of fluctuating resources on life history: patterns of allocation and plasticity in female guppies. Ecology, 74, 2011-2019. https://doi.org/10.2307/1940844

Riechert, S. E., \& Hedrick, A. V. (1993). A test for correlations among fitness-linked behavioural traits in the spider Agelenopsis aperta (Araneae, Agelenidae). Animal Behaviour, 46, 669-675. https://doi. org/10.1006/anbe.1993.1243

Rutz, C., \& St Clair, J. J. H. (2012). The evolutionary origins and ecological context of tool use in New Caledonian crows. Behavioural Processes, 89, 153-165. https://doi.org/10.1016/j.beproc.2011.11.005

Sih, A., Bell, A., \& Johnson, J. C. (2004). Behavioral syndromes: an ecological and evolutionary overview. Trends in Ecology \& Evolution, 19, 372-378. https://doi.org/10.1016/j.tree.2004.04.009

Sih, A., \& Del Giudice, M. (2012). Linking behavioural syndromes and cognition: a behavioural ecology perspective. Philosophical Transactions of the Royal Society B: Biological Sciences, 367, 2762-2772. https://doi. org/10.1098/rstb.2012.0216

Smith, B. R., \& Blumstein, D. T. (2008). Fitness consequences of personality: a meta-analysis. Behavioral Ecology, 19, 448-455. https://doi. org/10.1093/beheco/arm144

Smith, B. R., \& Blumstein, D. T. (2010). Behavioral types as predictors of survival in Trinidadian guppies (Poecilia reticulata). Behavioral Ecology, 21, 919-926. https://doi.org/10.1093/beheco/arq084

Team, R. D. C. (2015). R: A language and environment for statistical computing. Vienna, Austria: R Foundation for Statistical Computing.

Templeton, J. J., \& Giraldeau, L.-A. (1995). Patch assessment in foraging flocks of European starlings: evidence for the use of public information. Behavioral Ecology, 6, 65-72. https://doi.org/10.1093/beheco/6.1.65

Trompf, L., \& Brown, C. (2014). Personality affects learning and tradeoffs between private and social information in guppies, Poecilia reticulata. Animal Behaviour, 88, 99-106. https://doi.org/10.1016/j. anbehav.2013.11.022 
van Bergen, Y., Coolen, I., \& Laland, K. N. (2004). Nine-spined sticklebacks exploit the most reliable source when public and private information conflict. Proceedings of the Royal Society B: Biological Sciences, 271, 957. https://doi.org/10.1098/rspb.2004.2684

Verbeek, M. E., Boon, A., \& Drent, P. J. (1996). Exploration, aggressive behaviour and dominance in pair-wise confrontations of juvenile male great tits. Behaviour, 133, 945-963. https://doi.org/10.1163/156853996 X00314

Verbeek, M. E., Drent, P. J., \& Wiepkema, P. R. (1994). Consistent individual differences in early exploratory behaviour of male great tits. Animal Behaviour, 48, 1113-1121. https://doi.org/10.1006/anbe.1994.1344

Webster, S. J., \& Lefebvre, L. (2001). Problem solving and neophobia in a columbiform-passeriform assemblage in Barbados. Animal Behaviour, 62, 23-32. https://doi.org/10.1006/anbe. 2000.1725

Williams, S. E., \& Hoffman, E. A. (2009). Minimizing genetic adaptation in captive breeding programs: a review. Biological Conservation, 142, 2388-2400. https://doi.org/10.1016/j.biocon.2009.05.034

\section{SUPPORTING INFORMATION}

Additional supporting information may be found online in the Supporting Information section at the end of the article.

How to cite this article: Berdal MA, Rosenqvist G, Wright J. Innovation as part of a wider behavioural syndrome in the guppy: The effect of sex and body size. Ethology. 2018;124:760-772. https://doi.org/10.1111/eth.12810 